\title{
Analog capacitance ROM with IGFET bucket-brigade shift register
}

\author{
Bruun, Erik; Olesen, Ole
}

Published in:

I E E E Journal of Solid State Circuits

Publication date:

1975

Document Version

Publisher's PDF, also known as Version of record

Link back to DTU Orbit

Citation (APA):

Bruun, E., \& Olesen, O. (1975). Analog capacitance ROM with IGFET bucket-brigade shift register. I E E E Journal of Solid State Circuits, 10(1), 55-59.

\section{General rights}

Copyright and moral rights for the publications made accessible in the public portal are retained by the authors and/or other copyright owners and it is a condition of accessing publications that users recognise and abide by the legal requirements associated with these rights.

- Users may download and print one copy of any publication from the public portal for the purpose of private study or research.

- You may not further distribute the material or use it for any profit-making activity or commercial gain

- You may freely distribute the URL identifying the publication in the public portal

If you believe that this document breaches copyright please contact us providing details, and we will remove access to the work immediately and investigate your claim. 


\title{
Analog Capacitance ROM with IGFET Bucket-Brigade Shift Register
}

\author{
ERIK BRUUN, MEMBER, IEEE, AND OLE OLESEN
}

\begin{abstract}
A new type of monolithic analog read-only memory (AROM) is described. It consists of a memory element and associated on-chip readout circuitry. The memory can be used for storing sample values of time-varying analog signals. The memory element is a matrix of MOS capacitors, preprogrammed in size by a special mask. The readout element is a bucket-brigade (BB) shift register with parallel input and serial output. A test circuit that permits investigation of different principles of information transfer from capacitance matrix to shift register has been developed.
\end{abstract}

\section{INTRODUCTION}

$\mathrm{D}$ URING the recent years a number of charge-transfer devices have been developed [1]-[3], and some interesting applications as analog delay lines, image sensors, and monolithic transversal filters have emerged from these new devices [1], [4]. In this paper we describe a special kind of analog read-only memory (AROM) which utilizes a bucket-brigade device (BBD) [1] as the readout element.

The basic principle of the AROM is illustrated in Fig. 1. From a store consisting of a matrix of mask-programmed MOS capacitors a signal of $n$ samples is transferred in parallel to an analog shift register. By clocking the shift register, the signal samples are then read out at the serial output of the register.

In the store, each row of capacitors represents a signal with $n$ samples. Each sample value is represented by the size of a MOS capacitor. The store contains $m$ different signals ( $m$ rows in the matrix) each of which can be transferred to the BBD analog shift register independent of the others.

In the $\mathrm{BBD}$ information is represented by a signal charge on every second capacitor. The remaining capacitors in the BBD contain a reference charge. The transfer of information from the capacitance matrix to the BBD is therefore a transfer of charge between capacitors. The connection between the capacitors is established by a row of MOS transistors, one for each capacitor. After transfer to the BBD every second capacitor $C$ in the BBD must contain a charge which is a function of the size of the capacitor $C_{j}$ in the store with which charge exchange has taken place. This result can be obtained either by a transfer of charge from $C_{j}$ to $C$ or by a charge transfer from $C$ to $C_{j}$. The two possibilities will be discussed separately in the next section.

\section{Circuit Configuration and Operation}

Fig. 2 shows a complete circuit diagram of the test circuit for the AROM. The figure will be referred to in the following explanation of the circuit operation. The circuit offers the

Manuscript received July 18, 1974; revised September 19, 1974.

The authors are with the Laboratory for Semiconductor Devices, Technical University of Denmark, Lyngby, Denmark.

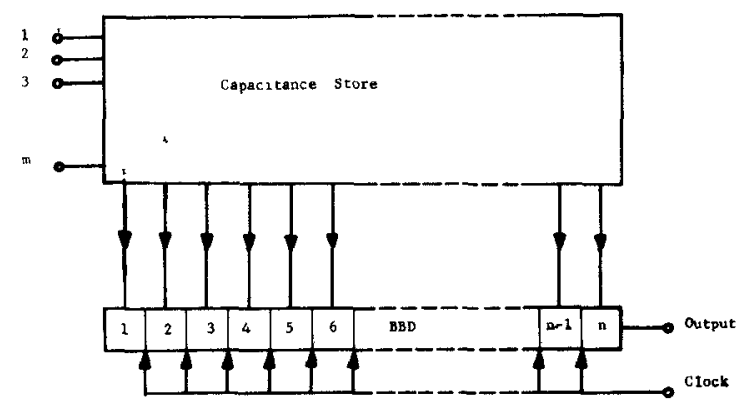

Fig. 1. Block diagram of the analog read-only memory (AROM).

possibility of testing different principles of charge transfer between capacitance matrix and BBD.

The circuits consist of a BBD with 92 stages and a capacitance matrix, each row of which contains 92 capacitors. In the particular test layout the number of rows is only 3 . With each capacitor in the matrix is associated a MOS transistor that establishes the connection to the BBD. Also contained in the circuit is a row of inhibit transistors and a row of clear transistors. These are only used for clearing the matrix of charge, and in the explanation of the charge transfer between matrix and BBD the inhibit transistors are assumed to be conducting while the clear transistors are nonconducting.

\section{A. Charge Transfer from Capacitance Row to BBD}

In our experiment the third row in the matrix is used for investigation of charge transfer from capacitance row to BBD.

To begin with, the capacitors $C$ in the BBD are assumed to have zero voltage. The other capacitors $C^{\prime}$ in the BBD are charged to the voltage $U_{0}-V_{T}$. $U_{0}$ is the amplitude of the clock pulses for the BBD and $V_{T}$ is the threshold voltage of the MOS transistors.

A reference pulse of amplitude $V_{\text {ref }}$ is applied to all capacitors $C_{31}$ to $C_{392}$ and a gatepulse $V_{g_{3}}$ is applied to the associated transistors $T_{31}$ to $T_{392}$. Each transistor in the third row then has $V_{d s}=V_{\text {ref }}$ and $V_{g s}=V_{g 3}$. If $V_{g s}>V_{T}$, the transistors will conduct and a current will charge $C$ until the source voltage $V_{s}$ reaches a value $V_{j}$. $V_{j}$ is the $j$ th sample value of the analog signal. The current flow will continue until either $V_{g s}=V_{T}$ or $V_{d s}=0 . \quad V_{g s}=V_{T}$ implies that $V_{S}=V_{g 3}-V_{T}$, giving the same voltage on all capacitors $C$ in the BBD, and is consequently of no interest. $V_{g s}$ must have a value that ensures $V_{g s}>V_{T}$. In this case the transfer stops when $V_{d s}=0$, and

$$
V_{d}=V_{s}=V_{j}=\frac{C_{j}}{C_{j}+C} V_{\mathrm{ref}}
$$




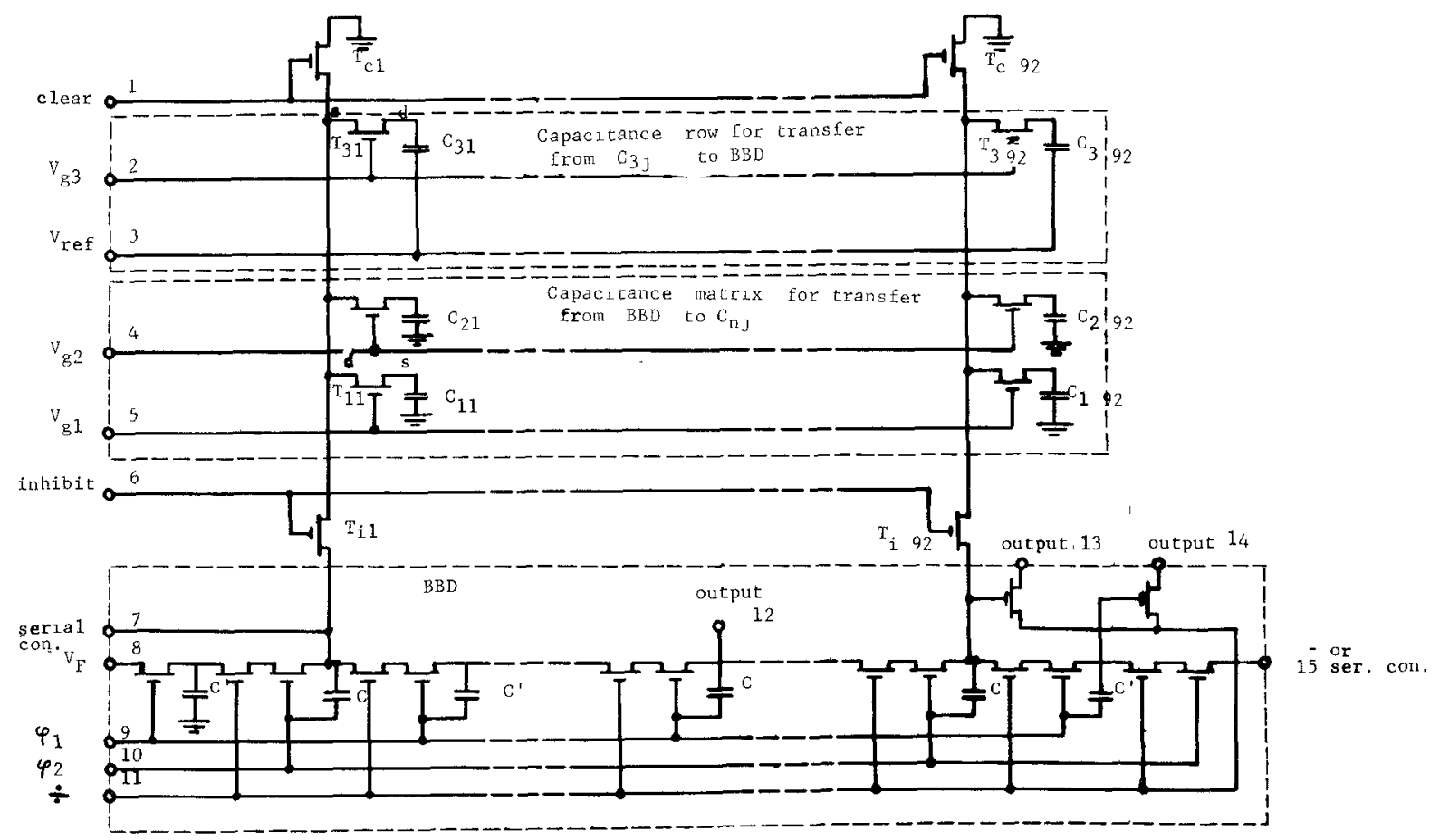

Fig. 2. Diagram of the AROM chip.

is obtained by a capacitive voltage division of $V_{\text {ref }}$. In order to ensure $V_{g s}>V_{T}, V_{g 3}$ must fulfill the following condition:

$$
V_{g 3}>\frac{C_{j \max }}{C_{j \max }+C} V_{\text {ref }}+V_{T} \text {. }
$$

$C_{j \max }$ is the maximum value of $C_{j}$ in the third capacitance row.

\section{B. Charge Transfer from BBD to Capacitance Row}

The two first rows in the matrix are used for investigation of charge transfer from BBD to capacitance row.

The capacitors $C^{\prime}$ in the BBD are again assumed to be charged to $U_{0}-V_{T}$. The capacitors $C$ are charged to a voltage $V_{F}$. This is obtained by applying $V_{F}$ at the serial input (terminal 8) of the BBD. The capacitors $\mathrm{C}_{1 j}$ and $C_{2 j}$ in the first and second row of the matrix are connected to ground.

To obtain a charge transfer from the capacitors $C$ in the BBD to the capacitors $C_{1 j}$ in the first row of the matrix, a pulse $V_{g}$ is applied to the gates of the transistors $T_{1 j}$, and, simultaneously, a clock pulse $\phi_{2}=U_{0}$ is applied to the capacitors $C$. The transistors $T_{1 j}$ obtain the initial condition $V_{d s}=U_{0}+$ $V_{F}$ and $V_{g s}=V_{g}$, and a current will flow and charge $C_{1 j}$ to a voltage $V_{s}$. Notice that in Fig. 2 source and drain of $T_{1 i}$ have been reversed compared to source and drain of $T_{3 j}$ because the direction of current flow has changed. The current flow will cease when $V_{d s}=0$ or $V_{g s}=V_{T}$.

1) Capacitive Voltage Division from $B B D$ to Capacitance Row: The first case, $V_{d s}=0$, gives a capacitive division of the voltage $U_{0}+V_{F}$ between $C$ and $C_{j}$, and the result is

$$
V_{s}=V_{d}=V_{j}=\frac{C}{C+C_{j}}\left(U_{0}+V_{F}\right) \text {. }
$$

Returning $V_{g}$ and $\phi_{2}$ to ground results in

$$
V_{j}=\frac{C}{C+C_{j}}\left(U_{0}+V_{F}\right)-U_{0} .
$$

The condition for obtaining capacitive voltage division is that $V_{g s}>V_{T}$ or

$$
V_{g}>\frac{C}{C_{j \min }+C}\left(U_{0}+V_{F}\right)+V_{T}
$$

where $C_{j \min }$ is the minimum capacitor in the row.

2) Bucket-Brigade (BB) Transfer from BBD to Capacitance Row: The second case, $V_{g s}=V_{T}$, gives $V_{s}=V_{g}-V_{T}$. The charge on $C_{j}$ then is

$$
Q_{j}=\left(V_{g}-V_{T}\right) C_{j}
$$

As this charge has been removed from $C$ the voltage on $C$ will be

$$
V_{j}=V_{F}+U_{0}-\frac{Q_{j}}{C}=V_{F}+U_{0}-\left(V_{g}-V_{T}\right) \frac{C_{j}}{C} .
$$

Returning $V_{g}$ and $\phi_{2}$ to ground results in

$$
V_{j}=V_{F}-\left(V_{g}-V_{T}\right) \frac{C_{j}}{C} .
$$

As this transfer mechanism is the same as the one that controls the charge transport between the capacitors in a BBD during a serial transfer it is called bucket-brigade (BB) transfer.

The condition for obtaining this kind of transfer is that $V_{d}$ is always greater than $V_{s}$, or 


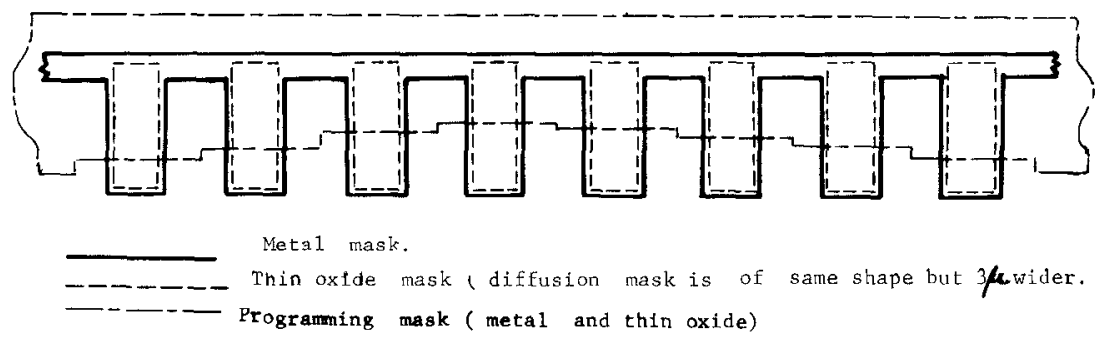

Fig. 3. Principle of mask for programming the capacitance values in the matrix.

$$
\begin{aligned}
& V_{F}+U_{0}-\left(V_{g}-V_{T}\right) \frac{C_{j}}{C}>V_{g}-V_{T} \\
& \Rightarrow V_{g}<\frac{C}{C_{j \max }+C}\left(U_{0}+V_{F}\right)+V_{T} .
\end{aligned}
$$

\section{Incomplete Charge Transport}

The calculated values of $V_{j}$ given in (1), (4), and (8) are limits obtained with infinite pulselengths. As the applied gate pulses have only a finite duration, a calculation of the dynamics of the charge transfer is necessary in order to give an estimate of the deviations from the simple expressions given above. Such a calculation has been carried out on the basis of a simple transistor model. The calculations are straightforward but rather tedious, so only the conclusion will be given here: it appears that the capacitive voltage division is approximately ten times faster than the BB transfer, but in both cases the transfer is so fast that the deviation of $V_{j}$ from the values calculated from the simple expressions is less than 1 percent for pulselengths greater than $10 \mu \mathrm{s}$, which is the smallest clock pulselength that gives satisfactory operation of the BBD shift register. Further, it should be noticed that only differences in the deviations from the ideal sample values will be a source of signal distortion. Equal errors only give a de level shift. Finally, it can be concluded that (1), (4), and (8) can be used without modifications for incomplete charge transfer.

\section{Clearing of the Capacitance Matrix}

After charge transfer, the capacitors in the matrix will contain a charge that has to be removed before the next charge transfer can take place. To remove the charge from a capacitor $C_{k j}$ the $p$ diffusion side of $C_{k j}$ has to be grounded. This can only be done through the associated transistor $T_{k j}$. A gate pulse opens $T_{k j}$, and the $j$ th column in the matrix is grounded by applying a gate pulse to the clear transistor $T_{c j}$ (index $c$ for clear). To prevent the capacitors $C$ in the BBD from being discharged, a row of transistors $T_{i j}$ (index $i$ for inhibit) is placed between the matrix and the BBD.

\section{Design Objectives}

\section{A. Layout}

The BBD is implemented with tetrode transistors in order to obtain the best transport parameter $\alpha$. The geometry is standard as shown in [5]. The size of the capacitors in the
BBD is approximately $1.6 \mathrm{pF}(60 \mu \mathrm{m}$ by $80 \mu \mathrm{m})$. Sourcefollower output buffers are provided at the serial output of the BBD.

The capacitance matrix is made with the same $p$ diffusion area for all capacitors, thus obtaining the same parasitic capacitance to substrate from all memory elements. The programming of capacitor size is done by a special mask which is used in both the thin oxide step and the aluminum step. The principle is shown in Fig. 3. Parts of the diffused areas do not have thin oxide and are not covered by aluminum. The use of a special mask for defining the capacitor values greatly facilitates reprogramming. In the test circuit, two rows (row 1 and row 2) have been programmed for BB transfer of charge from the shift register to the capacitor row. The encoded signals are a sine wave and a triangular wave, respectively. The third row has been programmed for capacitive voltage division and is encoded to generate a triangular wave. It can be used for investigating voltage division both from the capacitance row to the $\mathrm{BBD}$ and vice versa, since a signal sample $V_{j}$ obtained in the BBD after charge transfer shows the same dependence on $C_{j}$ in the two cases. This can be seen by rewriting (4):

$$
V_{j}=V_{F}-\frac{C_{j}}{C+C_{j}}\left(U_{0}+V_{F}\right) .
$$

When comparing this expression to (1) it is seen that in both cases the signal waveform is determined by $C_{j} /\left(C+C_{j}\right)$.

The maximum size of the matrix capacitors is approximately $0.85 \mathrm{pF}(32 \mu \mathrm{m}$ by $79 \mu \mathrm{m})$. The accuracy which can be obtained depends on the tracking of the various capacitors and this is inherently good because of small tolerances on masks and small variations in oxide thickness within each chip.

In the calculation of the capacitor values $C_{j}$ all parasitic capacitances have been taken into consideration. The most important parasitic capacitances are the junction capacitances from the capacitors in the BBD to substrate and from the matrix capacitors to substrate. When these are taken into account, (1), (3), and (7) should be modified to

$$
\begin{gathered}
V_{d}=V_{s}=V_{j}=\frac{C_{i}}{C_{j}+C_{p j}+C+C_{p}} V_{\mathrm{ref}} \\
V_{j}=\frac{C}{C+C_{p}+C_{j}+C_{p j}}\left(U_{0}+V_{F}\right)
\end{gathered}
$$




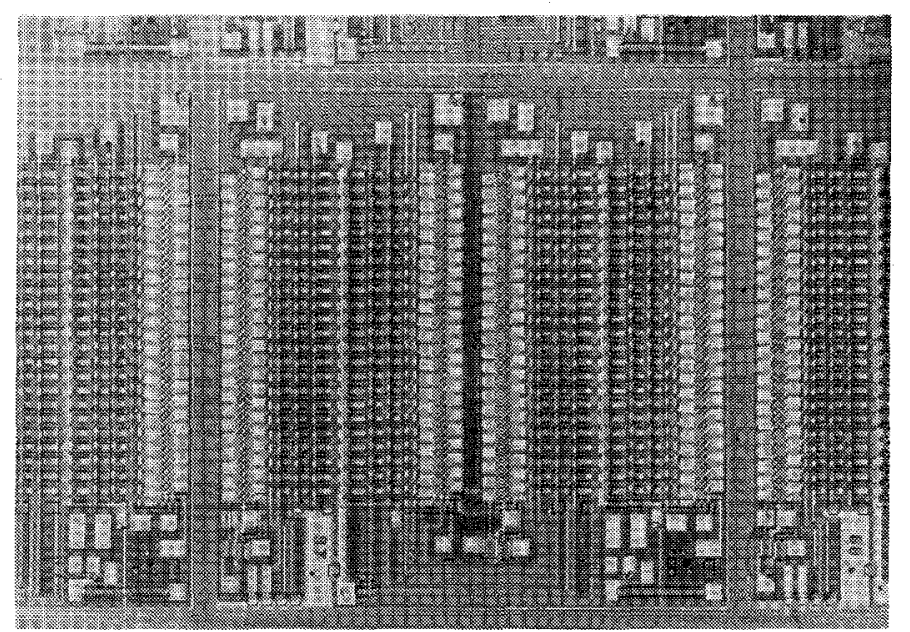

Fig. 4. AROM 3 by $3 \mathrm{~mm}^{2}$ test chip.

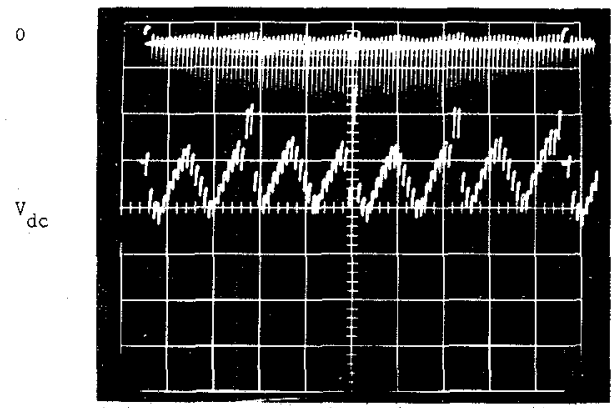

(a)

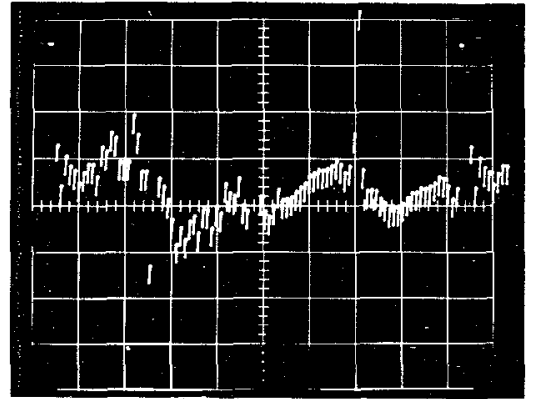

$\mathrm{U}_{\mathrm{O}}=-14 \mathrm{~V}$

$v_{F}=-4.0 \mathrm{~V}$

$f_{c}=20 \mathrm{kHz}$

horizontal

$0.5 \mathrm{~ms} / \mathrm{div}$.

vertical

$0.2 \mathrm{~V} / \mathrm{div}$.

Fig. 5. Output signals from BBD. (a) Voltage division from capacitance matrix to $\mathrm{BBD}$. (b) $\mathrm{BB}$ transfer from $\mathrm{BBD}$ to capacitance matrix.

$$
V_{j}=V_{F}+U_{0}-\left(V_{g}-V_{T}\right) \frac{C_{j}+C_{p j}}{C+C_{p}}
$$

where $C_{p}$ is the junction capacitance from the $\mathrm{p}$ diffusion side of $C$ to substrate, and $C_{p j}$ is the junction capacitance from the $\mathrm{p}$ diffusion side of $C_{j}$ to substrate. For the parasitic junction capacitors, the capacitance value per square unit will be approximately 10 percent of the desired oxide capacitances.

\section{B. Technology}

The AROM is produced using standard p-channel technology. The substrate is $5-9 \Omega \cdot \mathrm{cm}$ n-type $\langle 100\rangle$. Thin oxide $t_{\mathrm{ox}}=1000 \AA$. Threshold voltage $V_{T}=2.2 \mathrm{~V}$. (Calculated value.) The AROM circuit is shown in Fig. 4.

\section{Test Results}

Measurements on AROM circuits show wafer yields from 0 to 17 percent. Fig. 5 shows the triangular output signals. Voltage division gives the best result with no attenuation or DC level shift. Only an error from a noncompensated parasitic wiring capacitance is noticed. BB transfer from BBD to capacitance matrix may also give good results, but the output is dependent on the threshold voltage of transistor $T_{k j}$ as given in (7). If $V_{T}$ is not the same for all transistors in the row, noise will be imposed on the output signal as shown in Fig. 5(b).

To evaluate the output signal, peak voltages are measured from the source follower output of the BBD and divided by the source follower amplification. Fig. 6 shows the dependence of normalized peak output voltage versus clock amplitude $U_{0}$ and reference voltage $V_{\text {ref }}$ for $\mathrm{BB}$ transfer and voltage division, respectively. The results show the expected linear dependence close to the theoretical values. In Fig. 6(a) an extraordinary high value of $V_{T}$ gives a shift of the curve. In Fig. 6(b) a shift of the curve is also noticed. This is due to an offset in the clock voltages: instead of switching between 0 and $U_{0}, \phi_{1}$ and $\phi_{2}$ switches between $V_{\text {off }}$ and $U_{0}$, where $V_{\text {off }}$ is a dc offset voltage of approximately $2 \mathrm{~V}$.

\section{CONCLUSION}

It can be concluded that it is possible to construct an analog memory with a capacitance matrix and a BBD readout. Capacitive voltage division is the best method for information transport from matrix to $\mathrm{BBD}$ unless the threshold voltages 


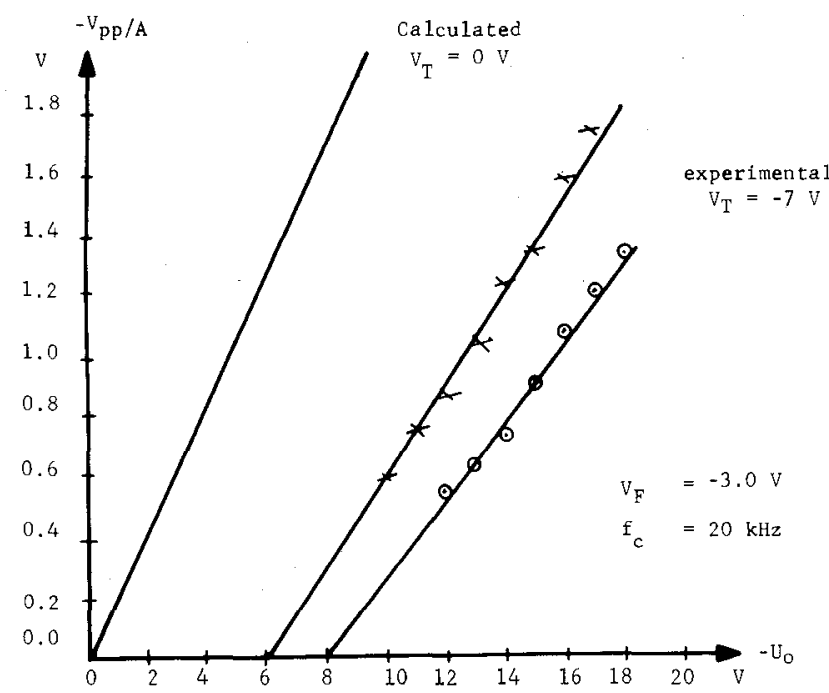

(a)

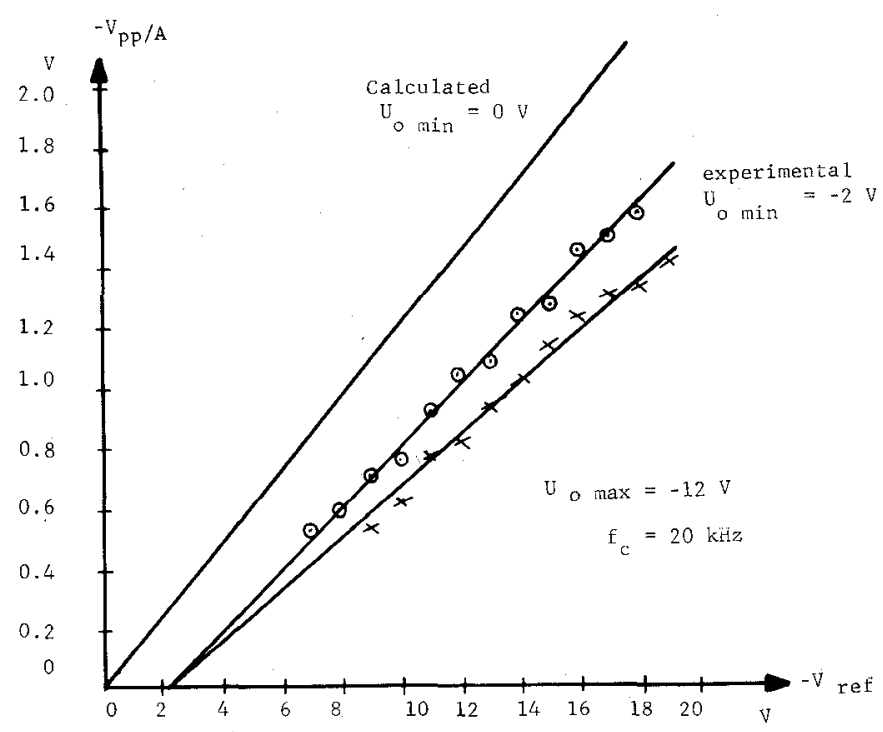

(b)

Fig. 6. Output peak voltage normalized with source follower amplification as (a) function of clock amplitude and (b) reference voltage $V_{\text {ref. }}$

show extremely low variation. Both theory and experiments show that charge transfer between matrix and BBD in all cases are faster than the serial transfer in the BBD. The limits in speed and clock frequency are the fundamental limits of the BBD.

If a $\mathrm{BBD}$ with 500 elements is required, which is the case in many applications, and standard tolerances are used the chip size will be 3 by $10 \mathrm{~mm}$ for only three rows. Thus if larger memory systems are needed hybrid solutions may be used.

\section{ACKNOWLEDGMENT}

The authors wish to thank Prof. G. Bruun for the idea of the AROM and for his constant interest in the work. Thanks are also due to D. Rebild and the laboratory staff for assistance in practical problems.

\section{REFERENCES}

[1] F. L. J. Sangster, "The bucket-brigade delay line, a shift register for analogue signals," Philips Tech. Rev., vol. 31, pp. 97-110, 1970.

[2] W. S. Boyle and G. E. Smith, "Charge-coupled semiconductor devices," Bell Syst. Tech. J., vol. 49, pp. 587-593, Apr. 1970.

[3] W. E. Engeler, J. J. Tiemann, and R. D. Baertsch, "The surfacecharge transistor," IEEE Trans. Electron Devices, vol. ED-18, pp. 1125-1136, Dec. 1971

[4] F. L. J. Sangster and K. Teer, "Bucket-brigade electronics-new possibilities for delay, time-axis conversion, and scanning," IEEE J. Solid-State Circuits, vol. SC-4, pp. 131-136, June 1969.

[5] F. L. J. Sangster, "Integrated bucket brigade delay line using MOStetrodes," Philips Tech. Rev., vol. 31, p. 266, 1970.

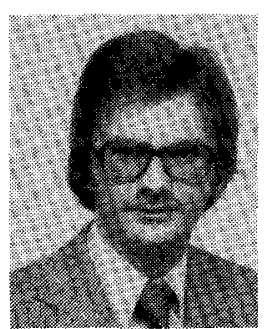

Erik Bruun (S'73-M'74) was born in Copenhagen, Denmark, on December 26, 1949. He received the M.S. degree in electrical engineering from the Technical University of Denmark, Lyngby, in 1974.

From January 1974 to September 1974 he was with Advanced Systems Department of Christian Rovsing $\mathrm{A} / \mathrm{S}$, where he was working on the development and construction of flighthardware and test equipment for a European satellite (Orbital Test Satellite, OTS). Since September 1974 he has been with the Laboratory for Semiconductor Devices at the Technical University of Denmark, where he is currently working towards the Ph.D. degree in the field of MNOS memories.

Mr. Bruun is a member of the Danish Engineering Society.

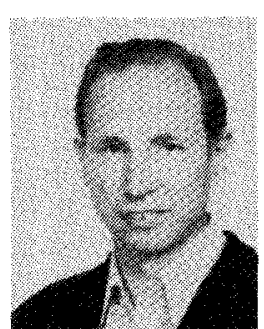

Ole Olesen was born in Fyen, Denmark, on February 16, 1932. He received the M.S. degree in electrical engineering and the Lic. Techn. degree (comparable to the Ph.D. degree) from the Technical University of Denmark, Lyngby, in 1958 and 1970 , respectively.

From 1958 to 1960 he worked in the electronic department of the Royal Danish Airforce on problems concerning airborne navigation equipment. From 1960 to 1967 he was a member of the Research and Teaching Staff of the Electronics Laboratory and from 1967 at the Laboratory for Semiconductor Devices both at the Technical University of Denmark. $\mathrm{He}$ is now Associate Professor. His research interests are in the area of charge transfer devices and semiconductor memories.

Dr. Olesen is a member of the Danish Engineering Society. 